\title{
Rationale for the Diabetic Retinopathy Clinical Research Network Treatment Protocol for Center- Involved Diabetic Macular Edema
}

\section{Citation}

Aiello, Lloyd Paul, Roy W. Beck, Neil M. Bressler, David J. Browning, K.V. Chalam, Matthew Davis, Frederick L. Ferris, et al. 2011. Rationale for the Diabetic Retinopathy Clinical Research Network Treatment Protocol for Center-Involved Diabetic Macular Edema. Ophthalmology 118, no. 12: e5-e14. doi:10.1016/j.ophtha.2011.09.058.

\section{Published Version}

doi:10.1016/j.ophtha.2011.09.058

\section{Permanent link}

http://nrs.harvard.edu/urn-3:HUL.InstRepos:35770525

\section{Terms of Use}

This article was downloaded from Harvard University's DASH repository, and is made available under the terms and conditions applicable to Other Posted Material, as set forth at http:// nrs.harvard.edu/urn-3:HUL.InstRepos:dash.current.terms-of-use\#LAA

\section{Share Your Story}

The Harvard community has made this article openly available.

Please share how this access benefits you. Submit a story.

Accessibility 
Published in final edited form as:

Ophthalmology. 2011 December ; 118(12): e5-e14. doi:10.1016/j.ophtha.2011.09.058.

\title{
Rationale for the Diabetic Retinopathy Clinical Research Network Treatment Protocol for Center-involved Diabetic Macular Edema
}

\author{
Diabetic Retinopathy Clinical Research Network ${ }^{\star}$, Lloyd Paul Aiello, MD, PhD ${ }^{1}$, Roy W \\ Beck, MD, PhD ${ }^{2}$, Neil M. Bressler, MD ${ }^{3}$, David J. Browning, MD, $\mathrm{PhD}^{4}$, KV Chalam, MD ${ }^{5}$, \\ Matthew Davis, MD ${ }^{6}$, Frederick L Ferris III, MD ${ }^{7}$, Adam Glassman ${ }^{2}$, Raj Maturi, MD ${ }^{8}$, Cynthia \\ R. Stockdale, $\mathbf{M S P H}^{2}$, and Trexler Topping, $\mathbf{M D}^{9}$ \\ ${ }^{1}$ Joslin Diabetes Center, Department of Ophthalmology, Harvard Medical School \\ 2Jaeb Center for Health Research \\ ${ }^{3}$ Wilmer Eye Institute, Johns Hopkins University School of Medicine \\ ${ }^{4}$ Charlotte Eye, Ear, Nose and Throat Assoc., PA \\ ${ }^{5}$ University of Florida College of Med., Department of Ophthalmology, Jacksonville Health \\ Science Center \\ ${ }^{6}$ University of Wisconsin, Department of Ophthalmology and Visual Sciences \\ ${ }^{7}$ National Eye Institutes and the National institutes of Health \\ ${ }^{8}$ Raj K. Maturi, M.D., P.C. \\ ${ }^{9}$ Ophthalmic Consultants of Boston
}

\begin{abstract}
Objective-Describe the underlying principles used to develop a web-based algorithm that incorporated intravitreal anti-vascular endothelial growth factor (anti-VEGF) treatment for diabetic macular edema (DME) in a Diabetic Retinopathy Clinical Research Network (DRCR.net) randomized clinical trial.
\end{abstract}

Design—Discussion of treatment protocol for DME.

Participants-Subjects with vision loss from DME involving the center of the macula.

\footnotetext{
(C) 2011 American Academy of Ophthalmology, Inc. Published by Elsevier Inc. All rights reserved.

Corresponding Author: Cynthia Stockdale, Jaeb Center for Health Research, 15310 Amberly Drive, Suite 350, Tampa, FL 33647; Phone: (813) 975-8690, Fax: (800) 816-7601, drcrnetstat5@ jaeb.org.

*The most recently published list of the Diabetic Retinopathy Clinical Research Network investigators and staff who participated in this study can be found at Ophthalmology 2010;117:1064-77.

An address for reprints will not be provided.
}

Financial Disclosure: The funding organization (National Institutes of Health) participated in oversight of the conduct of the study and review of the manuscript but not directly in the design or conduct of the study, nor in the collection, management, analysis, or interpretation of the data, or in the preparation of the manuscript. A complete list of all DRCR.net investigator financial disclosures can be found at www.drcr.net

Publisher's Disclaimer: This is a PDF file of an unedited manuscript that has been accepted for publication. As a service to our customers we are providing this early version of the manuscript. The manuscript will undergo copyediting, typesetting, and review of the resulting proof before it is published in its final citable form. Please note that during the production process errors may be discovered which could affect the content, and all legal disclaimers that apply to the journal pertain. 
Methods-The DRCR.net created an algorithm incorporating anti-VEGF injections in a comparative effectiveness randomized clinical trial evaluating intravitreal ranibizumab with prompt or deferred ( $\geq 24$ weeks) focal/grid laser in eyes with vision loss from center-involved DME. Results confirmed that intravitreal ranibizumab with prompt or deferred laser provides superior visual acuity outcomes, compared with prompt laser alone through at least 2 years. Duplication of this algorithm may not be practical for clinical practice. In order to share their opinion on how ophthalmologists might emulate the study protocol, participating DRCR.net investigators developed guidelines based on the algorithm's underlying rationale.

Main Outcome Measures-Clinical guidelines based on a DRCR.net protocol.

Results-The treatment protocol required real time feedback from a web-based data entry system for intravitreal injections, focal/grid laser, and follow-up intervals. Guidance from this system indicated whether treatment was required or given at investigator discretion and when follow-up should be scheduled. Clinical treatment guidelines, based on the underlying clinical rationale of the DRCR.net protocol, include repeating treatment monthly as long as there is improvement in edema compared with the previous month, or until the retina is no longer thickened. If thickening recurs or worsens after discontinuing treatment, treatment is resumed.

Conclusions-Duplication of the approach used in the DRCR.net randomized clinical trial to treat DME involving the center of the macula with intravitreal ranibizumab may not be practical in clinical practice, but likely can be emulated based on an understanding of the underlying rationale for the study protocol. Inherent differences between a web-based treatment algorithm and a clinical approach may lead to differences in outcomes that are impossible to predict. The closer the clinical approach is to the algorithm used in the study, the more likely the outcomes will be similar to those published.

\section{Introduction}

A randomized clinical trial by the Diabetic Retinopathy Clinical Research Network (DRCR.net) found that ranibizumab therapy with prompt (within 3 to 10 days after the initial injection) or deferred (for at least 24 weeks after the initial injection) focal/grid laser provided better visual acuity outcomes than prompt laser alone (within 3 to 10 days after an initial sham injection) through 2 years of follow-up in eyes with center involved diabetic macular edema (DME) causing vision loss. ${ }^{1}$ A detailed retreatment algorithm was implemented using a DRCR.net web-based real time data entry system that offered retreatment and follow-up scheduling guidance to investigators. The specific details of the retreatment algorithm are included in the form of flowcharts published previously. ${ }^{1}$ The underlying clinical principles that led to the development of the algorithm are outlined in Figure 1 and explained in this report as a potential guide for providing this treatment as interpreted by the DRCR.net investigators. The footnotes to Figure 1 provide additional details as to how the DRCR.net defined many of the terms within the clinical trial.

Questions addressed by the treatment protocol, including how those questions might be addressed in clinical practice, and how those questions have been addressed by the DRCR.net clinical trial investigators are summarized in Table 1 and discussed below. It is likely that the closer a clinician adheres to the study protocol the closer the results will mirror those published by the DRCR.net. Modifications could result in better, same, or worse results and clinicians should consider this as they decide on their individual treatment approach. A case from the DRCR.net trial (Appendix 1, Fig 2A through 2E) is provided as an example of how the retreatment algorithm and follow-up visit schedule was employed within the trial. 


\section{Which Patients with DME Were Considered for Anti-Vascular Endothelial Growth Factor (anti-VEGF) Therapy?}

In the DRCR.net protocol, patients could be enrolled if they had evidence of DME involving the center of the macula, defined as central subfield thickening of at least $250 \mu \mathrm{m}$ (at least 2 standard deviations beyond average normal thickness) on optical coherence tomography (OCT) using a Stratus (Carl Zeiss Meditec, Dublin, CA) time domain device. The OCT thickening was confirmed via clinical examination to be from the DME and not from concomitant findings such as an epiretinal membrane. Patients were included in the DRCR.net protocol with center involved DME, with or without prior laser treatment for DME, and any level of diabetic retinopathy (mild to severe non-proliferative diabetic retinopathy, or proliferative diabetic retinopathy that was adequately treated with panretinal photocoagulation at the time of enrollment). Patients were excluded if they had a history of recent (within 4 months) serious cardiovascular or cerebrovascular events.

Since clinicians may under-diagnose mild macular thickening that otherwise would be identified by OCT, 2,3 OCT-measured thickening alone, in an eye in which clinical examination identifies diabetic retinopathy and an absence of other causes of macular edema may be sufficient criteria for applying the findings of the trial. This consideration assumes that the clinical examination may have missed the DME if the OCT was depicting the thickening of the central macula accurately. For the purposes of this paper, all OCT measurements and guidelines are based on the Stratus device and its norms.

Patients with visual acuity better than 20/32 were excluded from the DRCR.net study both because it seemed appropriate to first demonstrate that the treatment was effective prior to using it in eyes with good visual acuity and because such eyes were unlikely to be able to reach the clinically relevant study outcomes such as a more than 10 or 15 letter improvement in visual acuity from baseline. Also of note, visual acuity in these studies is measured with high contrast letters (Electronic-Early Treatment Diabetic Retinopathy Study Visual Acuity Test) following a protocol-defined refraction. Typically visual acuities obtained using these standardized procedures and equipment tend to be better than Snellen visual acuities obtained in a standard clinical practice. ${ }^{4}$ Individual judgment would have to be used to decide whether eyes with central DME, but visual acuity better than 20/32 in clinical practice, should receive intravitreal anti-VEGF injections.

\section{What Intravitreal Injection Technique Is Used?}

Many different approaches to intravitreal injection have been proposed. ${ }^{5}$ For those wanting to emulate the DRCR.net intravitreal injection protocol, the specifics are as follows. Topical povidone-iodine, a sterile lid speculum, and topical anesthetic at the time of injection are required (Table 2). However, topical antibiotics before, on the day of, or prescribed to the patient after the injection, are not required, neither are sterile gloves, nor a drape. To avoid treatment of the wrong eye, the eye that is to receive an intravitreal injection is marked and topical anesthetic is applied to that eye. A sterile eyelid speculum is placed to stabilize the eyelids. Povidone iodine is applied to the conjunctiva directly over and surrounding the intended injection site, allowing time for the povidone iodine to dry before injection. Sterile calipers or the blunt end of a sterile syringe with a diameter equal to approximately $4.0 \mathrm{~mm}$ can be used to locate the position of the injection site $3.0 \mathrm{~mm}$ to $4.0 \mathrm{~mm}$ posterior to the limbus. The proper volume of drug to be injected is prepared by drawing $0.2 \mathrm{~mL}$ of ranibizumab into a sterile syringe using a sterile 19-gauge filter needle. The 19-gauge needle is removed and a sterile 30 -gauge needle is placed onto the syringe. With the needle cap removed, fluid is expelled until the plunger is advanced to $50 \mu \mathrm{L}(0.05 \mathrm{~mL})$. The drug is injected into the vitreous cavity with the needle pointing toward the optic nerve via the pars plana. After the injection, the lid speculum is removed. Measurement of intraocular pressure 
is not required after the injection. Measurement of gross visual acuity or direct visualization of the fundus confirms retinal artery perfusion. Topical antibiotics can be provided at the discretion of the treating ophthalmologist. As of February 2011, topical antibiotics after injection have been utilized in approximately $60 \%$ of all study ranibizumab injections in two concurrent DRCR.net Laser-Ranibizumab-Triamcinolone DRCR.net randomized trials involving intravitreal injections. ${ }^{1,6}$ Using this treatment protocol resulted in 5 cases of endophthalmitis after 6,251 ranibizumab injections (as of February 2011). Thus far, all five cases of endophthalmitis have occurred in eyes using topical antibiotics. No cases of endophthalmitis have occurred among the approximately 2,500 ranibizumab injections given in the absence of topical antibiotic drops either before the injection or prescribed to the patient after the injection. While it is unknown whether modifications to this technique would provide greater or lesser patient comfort, or increase or decrease the risk of endophthalmitis, the risk of endophthalmitis following this protocol and within the DRCR.net is low but not zero (approximately $0.8 \%$ of subjects and $0.08 \%$ of injections) when treating DME.

\section{How Frequently Were Protocol Participants Seen and Treated After Initiating Ranibizumab Therapy?}

In the DRCR.net protocol, follow-up was at 4-week intervals through the first year. Fourweek intervals were chosen because pharmacokinetic data suggest that intravitreal ranibizumab is unlikely to remain within the vitreous in a concentration sufficient to result in any further improvement between 21 and 28 days after injection. ${ }^{1}$ The DRCR.net protocol required 4 to 6 initial injections before treatment could be withheld. It is unknown what effect using an "as needed" approach would have had on injection frequency or outcomes. However, after four initial ranibizumab injections (Table 3) approximately $50 \%$ of eyes attained 20/20 visual acuity or had a central subfield thickness of less than $250 \mu \mathrm{m}$ (defined as "success", after which further injections were at investigator discretion). Seventeen percent of the eyes that had not achieved success after four injections did achieve success after five injections; $15 \%$ of the eyes that had not achieve success after five injections did achieve success after six injections.

After the 4 to 6 required injections in the DRCR.net protocol, retreatment continued every 4 weeks until one of three outcomes occurred: 1) "success" (defined above); 2) there had been "no further improvement" defined as $<10 \%$ decrease in central subfield thickness and $<5$ letter increase in visual acuity since the most recent injection, and, in the opinion of the treating ophthalmologist, it seemed unlikely that additional treatment would provide any further benefit; or 3) the result of treatment was considered a treatment "failure", defined as definite worsening despite treatment, or serious side effects associated with treatment precluding additional treatment.

If treatment was withheld because there was "no further improvement", a follow-up visit was scheduled 4 weeks later to see if the persistent edema worsened. If the edema worsened at the next follow-up exam, an injection was recommended, but the decision to re-inject was ultimately at investigator discretion.

Definite worsening, or treatment failure, included persistent edema along with 10 or more letter worsening from baseline at any visit, or, after at least 1 year of treatment, there was no improvement from baseline in central subfield thickness or visual acuity in the setting of "complete" laser. "Complete" laser was defined as direct treatment to all microaneurysms within areas of macular edema and grid treatment already applied to all other areas of macular edema. Once "failure" criteria were met, anti-VEGF treatment could be discontinued and any alternative treatment (such as intravitreal corticosteroids) could be performed. 
Through 48 weeks, follow-up visits were every 4 weeks regardless of whether an injection was given. Starting at the 52-week visit, if treatment was withheld due to "success" or "no further improvement" (as described above) for at least 3 consecutive visits, follow-up then could be extended to 8 weeks. At that point, if the edema did not recur or worsen and no injection was given, follow-up could be extended to 16 weeks. If "failure" was met, followup also could be extended immediately to 16 weeks. Only $1 \%$ of eyes assigned to ranibizumab met "failure" criteria through the 48 -week visit.

The total number of treatments and visits during the first two years of following this protocol in the DRCR.net study are summarized in Table 4. In the first 20 weeks, a median of 6 injections were given in the ranibizumab groups. Between the 24-week and 48-week visits, an additional median of 3 injections were given in both ranibizumab groups. In the second year, an additional median of 2 (ranibizumab+prompt laser) or 3 (ranibizumab + deferred laser) injections were given. A median of 2 and 0 lasers were given in the first year in the ranibizumab+prompt laser and ranibizumab+deferred laser group, respectively, and a median of 0 lasers in both groups in the second year. In the first year, participants were required to complete visits every 4 weeks per protocol. Once follow-up could be extended in the second year, the median total number of visits was 8 and 10 in the ranibizumab+prompt laser and ranibizumab+deferred laser group, respectively.

Since duplication of this approach may not be practical in clinical practice, Figure 1, and the second column of Table 1, provide guidelines suggested by the DRCR.net investigators to apply this treatment protocol to clinical practice without a web-based real time data entry system. These guidelines include repeating treatment monthly as long as there is improvement in edema compared with the previous month, or until the retina is no longer thickened.

\section{Once Treatment is Withheld, When Was Treatment Resumed?}

In the DRCR.net protocol, resuming treatment after it had been withheld because of "success" or "no further improvement" was at investigator discretion. Treatment always was recommended if edema recurred or increased after discontinuing injections. Once antiVEGF therapy was resumed, follow-up returned to every 4 weeks until treatment was again withheld at 3 consecutive visits for success or no further improvement, at which point follow-up could be re-extended as described above.

Figure 1 and the second column of Table 1 provide guidelines suggested by the DRCR.net investigators which may assist an ophthalmologist to determine when treatment might be resumed after anti-VEGF is withheld based on the underlying rationale of the DRCR.net protocol for DME. Specifically, if thickening recurs or worsens after discontinuing treatment, then treatment is resumed. Once treatment is resumed, monthly follow-up also might be resumed until success criteria are met or additional treatment is judged unlikely to be beneficial because of lack of improvement compared with the previous visit. If thickening does not recur or worsen after discontinuing treatment, then follow-up can be doubled to 2 months. If thickening recurs or worsens after 2 months, then treatment is resumed, and if thickening does not recur or worsen after 2 months, then follow-up can be doubled again to every 4 months until thickening recurs or worsens.

\section{How Might Focal/Grid Laser Be Incorporated Within Anti-VEGF Therapy for DME?}

In the DRCR.net protocol, focal/grid laser was assigned at random at baseline to be given either promptly (within 3 to 10 days) after initiation of intravitreal ranibizumab or deferred for at least 24 weeks. In the "deferred" focal/grid laser group, laser treatment was deferred 
for at least 24 weeks. Treatment was only initiated in eyes with persistent DME that had "no further improvement" (as defined above) after the two most recent consecutive injections.

The DRCR.net focal/grid laser protocol is a modification of the ETDRS protocol (Table 5). ${ }^{7}$ In the first year, approximately $70 \%$ of eyes in the deferred laser group improved with injections alone and did not require laser treatment. It is unknown whether prompt or deferred laser is preferred at this time, although this question is being evaluated with continued follow-up.

Once focal/grid laser is initiated, retreatment with focal/grid laser was considered in the trial if 3 criteria were met (regardless of whether an injection was given), and these criteria are recommended for consideration in clinical practice as follows: 1) edema is threatening or involving the center of the macula (for the trial, this was defined specifically as OCT central subfield thickness $\geq 250 \mu \mathrm{m}$, edema within $500 \mu \mathrm{m}$ of the center of the macula, edema associated with lipid within $500 \mu \mathrm{m}$ of the center of the macula, or edema $\geq 1$ disc area within 1 disc area of the center of the macula); 2) laser treatment is not yet "complete"; and 3 ) it has been at least 13 weeks since previous focal/grid laser treatment.

\section{Example of DME Treatment Following the DRCR.net Retreatment Algorithm}

An example of DME treatment following the DRCR.net retreatment algorithm is provided in Appendix 1, and Figures 2a through 2e.

\section{Summary}

A randomized clinical trial by the DRCR.net found that intravitreal ranibizumab therapy with either prompt or deferred (for at least 24 weeks) focal/grid laser provided better visual acuity outcomes compared with prompt laser alone through 2 years in eyes with vision impairment from center-involved DME. These outcomes have been confirmed in one smaller study utilizing bevacizumab with a slightly different retreatment algorithm and three other Phase 3 randomized clinical trials utilizing ranibizumab with other retreatment algorithms. ${ }^{8-10}$

The underlying rationale of the DRCR.net treatment algorithm for DME with intravitreal ranibizumab therapy requires monthly injections until an eye reaches "success" (the macular edema resolves or vision reached 20/20 or better); or until additional treatment is judged unlikely to be beneficial because of "no further improvement" compared with the previous visit(s) (edema improved after initiation of treatment, but eventually stabilized without reaching "success"); or an eye meets "failure" criteria (edema worsened or remained unaffected by treatment). Once ranibizumab is withheld, treatment could be resumed if macular edema recurs or worsens. If treatment is withheld and edema does not recur or worsen, the follow-up time could be doubled and if edema still does not recur or worsen, follow-up could be doubled again according to the study protocol.

In the DRCR.net study evaluating ranibizumab for DME, focal/grid laser was combined initially with anti-VEGF treatment or deferred for at least 6 months. After that, it was considered if macular edema persisted or was not improving despite anti-VEGF treatment. Additional focal/grid laser can be added as often as every 4 months.

The treatment benefit obtained following the DRCR.net protocol was achieved with standardized feedback and guidance for intravitreal injections, focal/grid laser, and followup intervals using a DRCR.net web-based real time data entry system. The guidance from this system indicated whether treatment was required or could be given at investigator discretion. Duplication of the approach used within the infrastructure of this clinical trial may not be practical in clinical practice, but can be emulated fairly closely based on an 
understanding of the underlying rationale for the study protocol. To what degree modifications to the DRCR.net protocol in the absence of the web-based computer data entry system will result in different outcomes is unknown. Because a web-based algorithm is not feasible in many clinical practices, following the underlying principles used to develop that algorithm as interpreted and explained by the DRCR.net investigators in this report, may be a practical clinical alternative.

\section{Acknowledgments}

Financial Support: Supported through a cooperative agreement from the National Eye Institute and the National Institute of Diabetes and Digestive and Kidney Diseases, National Institutes of Health, U.S. Department of Health and Human Services EY14231, EY14229, EY018817

\section{Appendix 1}

A 58 year old white man with Type 2 diabetes for the past 2 years and an $\mathrm{HgA} 1 \mathrm{c}$ of $5.7 \%$ presented with a visual acuity of 20/32 in the right eye and 20/40 in the left eye. Clinical examination showed diabetic macular edema involving the center of the macula in the right eye with an optical coherence tomography (OCT) central subfield thickness (CST) of $631 \mu \mathrm{m}$. His right eye was phakic. The right eye had no prior treatment for diabetic macular edema (DME). He was assigned randomly to ranibizumab+prompt focal/grid laser in the right eye and received intravitreal ranibizumab followed approximately 1 week later with focal/grid laser. At the 4-week visit, visual acuity improved to 20/25 and OCT CST improved to $419 \mu \mathrm{m}$ and intravitreal ranibizumab was repeated. At the 8 -week visit, visual acuity again improved to $20 / 20$ but the OCT CST was still thickened at $348 \mu \mathrm{m}$. Despite the $20 / 20$ visual acuity, intravitreal ranibizumab was repeated at the 8 - and 12-week visit, as was required by the protocol. At the 16-week visit, the visual acuity remained 20/20 but the OCT CST still was thickened at $332 \mu \mathrm{m}$. Intravitreal ranibizumab could be withheld at the 16-week visit and was. However, because the patient had been assigned to ranibizumab +prompt laser, and there still was abnormal thickening of the central subfield, focal/grid laser was repeated. At the 20-week visit, the visual acuity remained 20/20, but the OCT CST increased to $408 \mu \mathrm{m}$. Again, intravitreal ranibizumab was withheld because of the excellent visual acuity, despite the persistent central subfield thickening. There was no additional treatment until the 32-week visit when the visual acuity remained 20/20 but the OCT CSF remained abnormally thickened at $319 \mu \mathrm{m}$. Intravitreal ranibizumab given at investigator discretion and repeat focal/grid laser 1 week later were added. Intravitreal ranibizumab was repeated at the 40-week visit and by the 48-week visit the visual acuity was 20/25 and the OCT CSF was $316 \mu \mathrm{m}$. Intravitreal ranibizumab again was applied with focal/grid laser 1 week later. Four weeks later, at the 52-week visit, the visual acuity was 20/20 and the OCT CSF was $250 \mu \mathrm{m}$.

This patient is an example of substantial improvement in visual acuity by the 8-week visit which was sustained through 12 months, but a prolonged course of slow anatomic improvement was noted in which 3 additional focal/grid lasers and several additional intravitreal ranibizumab injections were given.

\section{References}

1. Elman MJ, Aiello LP, Beck RW, et al. Diabetic Retinopathy Clinical Research Network. Randomized trial evaluating ranibizumab plus prompt or deferred laser or triamcinolone plus prompt laser for diabetic macular edema. Ophthalmology. 2010; 117:1064-77. [PubMed: 20427088] 
2. Brown JC, Solomon SD, Bressler SB, et al. Detection of diabetic foveal edema: contact lens biomicroscopy compared with optical coherence tomography. Arch Ophthalmol. 2004; 122:330-5. [PubMed: 15006844]

3. Browning DJ, McOwen MD, Bowen RM Jr, O'Marah TL. Comparison of the clinical diagnosis of diabetic macular edema with diagnosis by optical coherence tomography. Ophthalmology. 2004; 111:712-5. [PubMed: 15051203]

4. Falkenstein IA, Cochran DE, Azen SP, et al. Comparison of visual acuity in macular degeneration patients measured with Snellen and Early Treatment Diabetic Retinopathy Study charts. Ophthalmology. 2008; 115:319-23. [PubMed: 17706288]

5. Aiello LP, Brucker AJ, Chang S, et al. Evolving guidelines for intravitreous injections. Retina. 2004; 24(suppl):S3-19. [PubMed: 15483476]

6. Diabetic Retinopathy Clinical Research Network. A randomized trial comparing intravitreal triamcinolone acetonide and focal/grid photocoagulation for diabetic macular edema. Ophthalmology. 2008; 115:1447-59. [PubMed: 18662829]

7. Writing Committee for the Diabetic Retinopathy Clinical Research Network. Comparison of the modified Early Treatment Diabetic Retinopathy Study and mild macular grid laser photocoagulation strategies for diabetic macular edema. Arch Ophthalmol. 2007; 125:469-80. [PubMed: 17420366]

8. Michaelides M, Kaines A, Hamilton RD, et al. A prospective randomized trial of intravitreal Bevacizumab or Laser Therapy in the management of diabetic macular edema (BOLT study): 12month data: report 2. Ophthalmology. 2010; 117:1078-86. [PubMed: 20416952]

9. Ranibizumab significantly more effective than standard of care in treating vision loss due to DME, a serious complication of diabetes [press release]. Basel Switzerland: Novartis Global; May 22. 2010 Available at: http://www.novartis.com/newsroom/media-releases/en/2010/1418121.shtml

10. Lucentis phase III study meets primary endpoint for improving vision in patients with diabetic macular edema (DME) [press release]. San Francisco, CA: Genentech; February 11. 2011 Available at: http://www.gene.com/gene/news/press-releases/display.do?method=detail=13247 


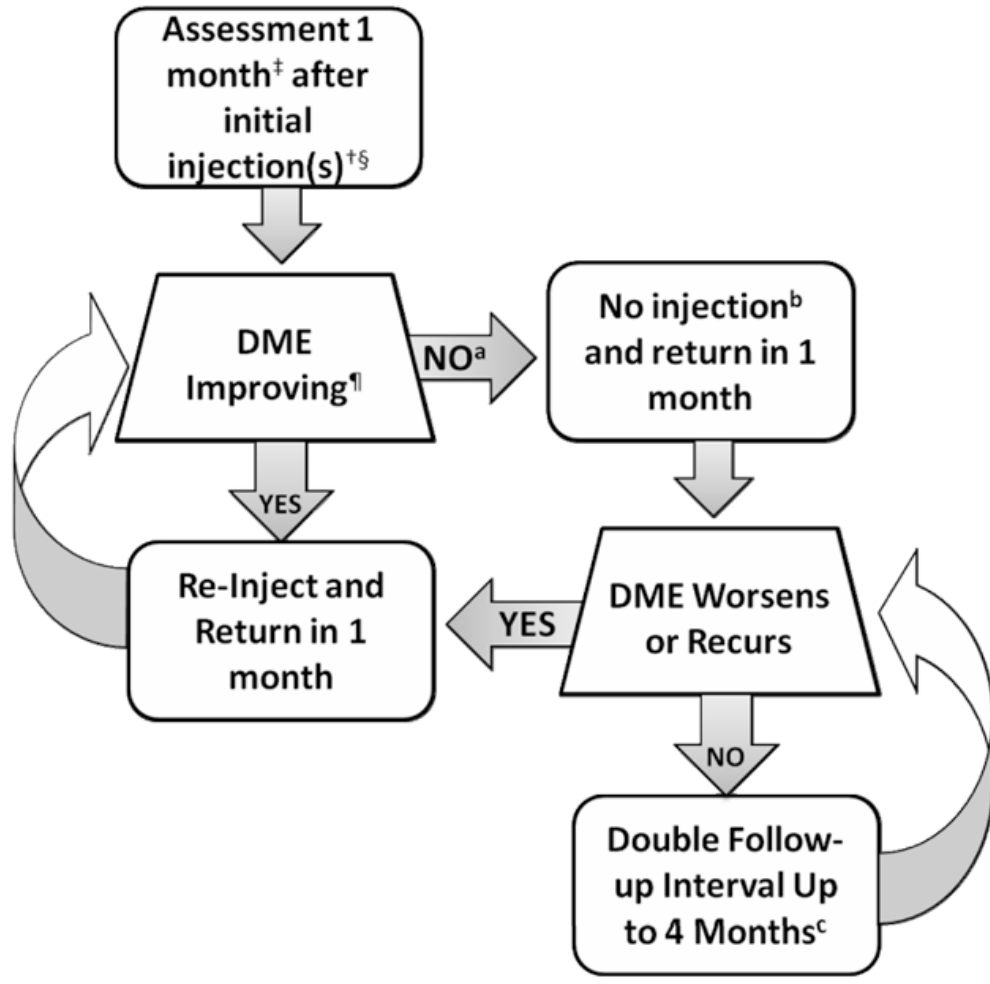

$\mp \quad$ In the DRCR.net study, 4-week, not 1-month, intervals were used.

$\S$ The DRCR.net study required 4 injections of intravitreal ranibizumab every 4 weeks initially; it is not known whether a different number of injections initially would have worked as well. DRCR. net also required 2 additional injections at months 5 and 6 if edema persisted and success had not been met, even in the absence of improvement

I Relevant details from the DRCR net study: 1) DRCR. net "improvement" on Zeiss Stratus OCT $>10 \%$ decrease in central subfield thickness. 2) Even if no longer improving on OCT, injections continued if visual acuity "improvement" (unless $20 / 20$ or better). 3) Visual acuity improvement defined as 5 or more letter increase on Electronic-Early Treatment Diabetic Retinopathy Study.

a In the DRCR.net study, if focal/grid laser was deferred at baseline, it was added at or after 24 weeks if edema still present and OCT central subfield and vision no longer improving

b In the DRCR net study, all patients received at least 4 injections 4 weeks apart. The decision to re-inject was at investigator discretion starting at 16 weeks for "success", defined as visual acuity better than 20/20 or OCT central subfield $<250 \mu \mathrm{m}$; starting at 24 weeks, re-injection also was at investigator discretion if no improvement in OCT central subfield or vision.

c The DRCR.net study continued follow-up every 4 weeks through the 52-week visit and did not permit extension of followup until after the 52 -week visit. If injection was withheld due to no improvement or success at 3 consecutive visits following the week 52 visit, follow-up interval was doubled to 8 weeks and then again to 16 weeks if still no change.

Figure 1.

Diabetic Retinopathy Clinical Research Network (DRCR.net) Rationale for Treatment and Follow-up of Center-Involved Diabetic Macular Edema (DME) with Anti-Vascular Endothelial Growth Factor Therapy. Only eyes with vision impairment (approximate Snellen equivalent 20/32 to 20/320) from DME were included. Optical Coherence Tomography=OCT. Additional terms utilized within the clinical trial are defined in the footnotes. 


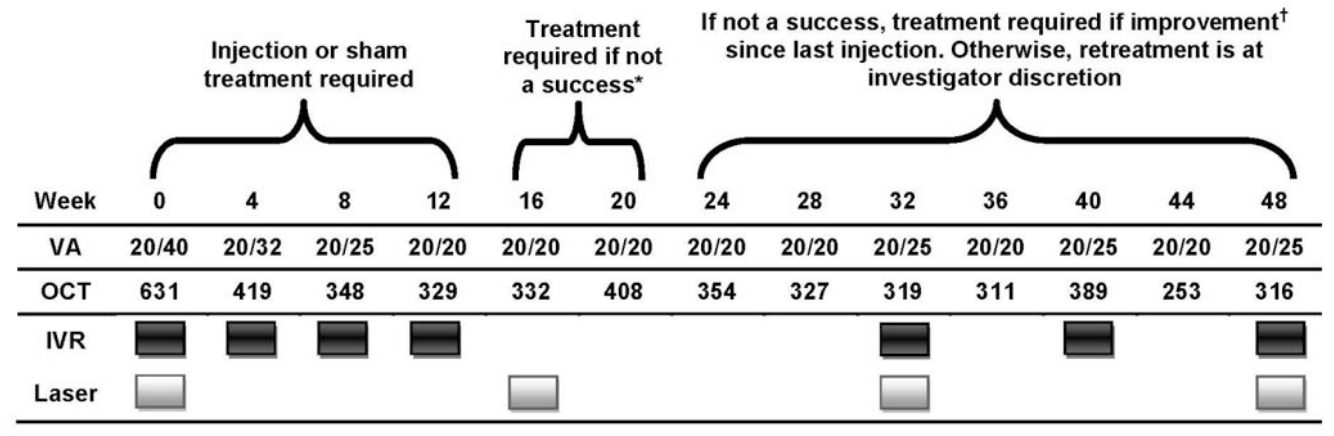

Ranibizumab

Injection Performed

Focal/grid Laser

Performed

* Success $=$ visual acuity letter score $\geq 84(\sim 20 / 20)$ or OCT CSF $<250 \mu \mathrm{m}$; retreatment at investigator discretion. $\dagger$ Improvement $=$ OCT central subfield thickness decreased by $\geq 10 \%$ or visual acuity letter score improved by $\geq 5$.

Extended

Extended

Follow-up to

Follow-up to

8 Weeks

16 Weeks

\begin{tabular}{|c|c|c|c|c|c|c|c|c|c|c|c|c|c|c|}
\hline Week & 52 & 56 & 60 & 64 & 68 & 72 & 76 & 80 & 84 & 88 & 92 & 96 & 100 & 104 \\
\hline VA & $20 / 20$ & $20 / 20$ & $20 / 20$ & $20 / 20$ & $20 / 20$ & $20 / 16$ & & $20 / 20$ & & & & $20 / 20$ & & $20 / 20$ \\
\hline OCT & 250 & 252 & 289 & 211 & 207 & 208 & & 211 & & & & 194 & & 215 \\
\hline
\end{tabular}

Laser

$\square$

Ranibizumab

Injection Performed

Focal/grid Laser

Performed 

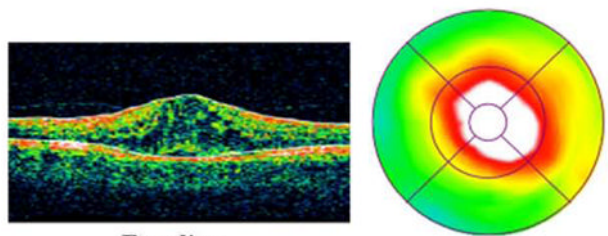

Baseline

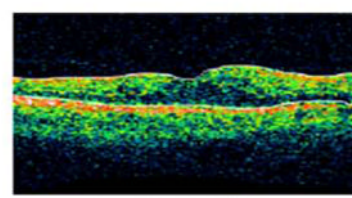

16-Week Visit

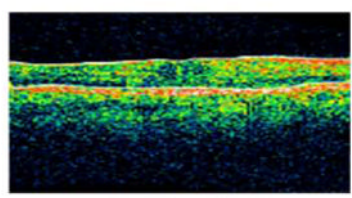

32-Week Visit

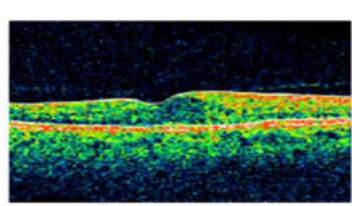

48-Week Visit
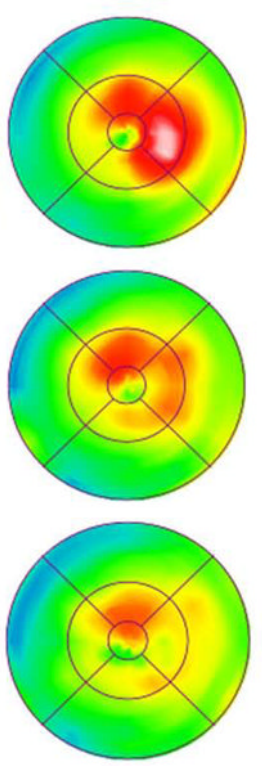

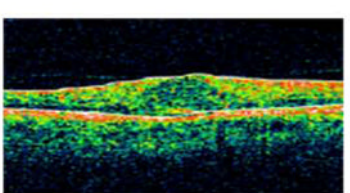

4-Week Visit

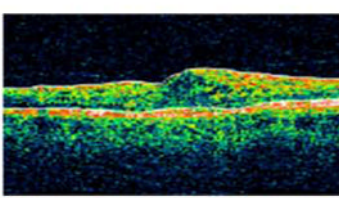

24-Week Visit

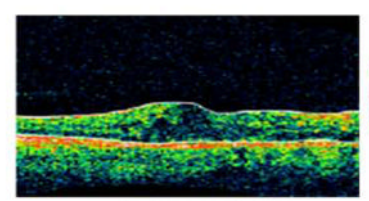

40-Week Visit

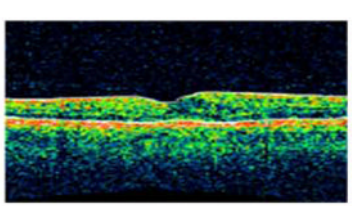

52-Week Visit
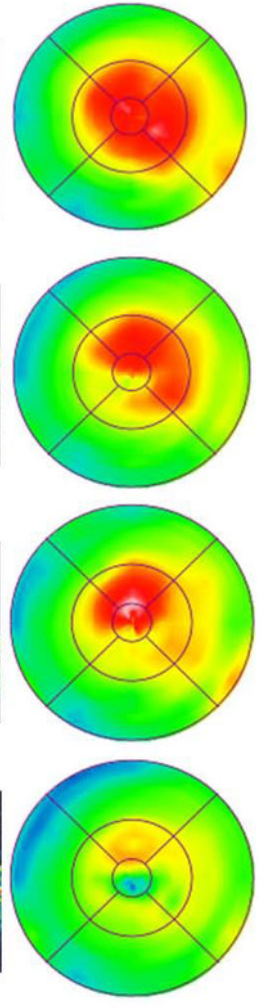

Ophthalmology. Author manuscript; available in PMC 2012 December 1. 
Figure 2.

Figure 2a. Case Example for Diabetic Retinopathy Clinical Research Network Treatment Algorithm - Year 1. VA= Visual Acuity, OCT= Optical Coherence Tomography, IVR= Intravitreal Ranibuzumab, $\mathrm{CSF}=$ Central Subfield

Figure 2b. Case Example for Diabetic Retinopathy Clinical Research Network Treatment Algorithm - Year 2. VA= Visual Acuity, OCT= Optical Coherence Tomography, IVR= Intravitreal Ranibuzumab

Figure 2c. Case Example Baseline Color Fundus Photograph

Figure 2d. Case Example OCT Cross-sectional Images and Retinal Thickness Maps by Visit.

Figure 2e. Case Example 52-Week Color Fundus Photograph 
Table 1

Diabetic Retinopathy Clinical Research Network Approach to Treating Diabetic Macular Edema

\begin{tabular}{|c|c|c|}
\hline Concept & Clinical Practice Considerations & DRCR.net Study \\
\hline $\begin{array}{l}\text { In which eyes with } \\
\text { DME should anti-VEGF } \\
\text { therapy be considered? }\end{array}$ & Eyes with edema involving the center of the macula & $\begin{array}{l}\text { Patients could be enrolled if they had edema } \\
\text { involving the center of the macula defined as } \\
\text { central subfield thickness } \geq 250 \mu \text { and vision } \\
\text { impairment defined as Snellen equivalent of } \\
20 / 32 \text { to } 20 / 320 \text {. }\end{array}$ \\
\hline $\begin{array}{l}\text { What treatment regimen } \\
\text { should be considered } \\
\text { after initiating therapy? }\end{array}$ & $\begin{array}{l}\text { A series of several monthly injections followed by continued } \\
\text { injections at monthly intervals until vision and edema are no } \\
\text { longer improving }{ }^{\dagger} \text { or can no longer improve (e.g. vision } 20 / 20 \\
\text { or better, or edema resolved) }\end{array}$ & $\begin{array}{l}4 \text { required injections of ranibizumab at monthly } \\
\text { intervals, followed by } 2 \text { injections at next } \\
\text { monthly intervals unless edema resolved or } \\
\text { vision improved to } 20 / 20 \text { or better }\end{array}$ \\
\hline $\begin{array}{l}\text { What treatment is } \\
\text { employed when the } \\
\text { DME no longer is } \\
\text { improving? }\end{array}$ & $\begin{array}{l}\text { Once an eye is no longer improving, focal/grid laser can be } \\
\text { added if there are areas which can be treated (untreated } \\
\text { microaneurysms within areas of thickened retina and other } \\
\text { areas of thickening without grid laser) and in this case } \\
\text { injections would continue. Injections may be withheld if } \\
\text { additional treatment at that visit seemed unlikely to provide } \\
\text { any further benefit. }\end{array}$ & $\begin{array}{l}\text { If no improvement from prior injection, decision } \\
\text { to re-inject was at investigator discretion. } \\
\text { Injections were recommended if there still was } \\
\text { edema to treat. }\end{array}$ \\
\hline $\begin{array}{l}\text { When should treatment } \\
\text { be resumed after it has } \\
\text { been withheld at a } \\
\text { particular visit? }\end{array}$ & $\begin{array}{l}\text { Injections may be resumed if edema recurs or worsens; } \\
\text { follow-up then returns to monthly intervals until injections can } \\
\text { be withheld without worsening or recurring edema. }\end{array}$ & $\begin{array}{l}\text { Re-injection was at the investigator's discretion } \\
\text { if edema recurred or worsened, but was } \\
\text { recommended if there was edema to treat; } \\
\text { follow-up then returned to 4-week intervals once } \\
\text { injections resumed }\end{array}$ \\
\hline $\begin{array}{l}\text { What follow-up is } \\
\text { employed when an } \\
\text { injection is not given } \\
\text { and the DME does not } \\
\text { recur or worsen at the } \\
\text { next follow-up visit? }\end{array}$ & $\begin{array}{l}\text { If injection is not given and edema does not recur or worsen, } \\
\text { follow-up may be doubled (up to } 4 \text { months or longer). }\end{array}$ & $\begin{array}{l}\text { Starting with the second year of treatment, if an } \\
\text { injection was withheld at } 3 \text { consecutive monthly } \\
\text { visits, the follow-up interval was extended to } 8 \\
\text { weeks, and then again to } 16 \text { weeks if treatment } \\
\text { was still not warranted at the } 8 \text { week visit }\end{array}$ \\
\hline $\begin{array}{l}\text { When should focal/grid } \\
\text { laser treatment be added } \\
\text { to intravitreal anti- } \\
\text { VEGF therapy for } \\
\text { DME? }\end{array}$ & $\begin{array}{l}\text { Focal/grid laser may be given either initially or deferred. If } \\
\text { deferred, laser may be added at any time if edema persists and } \\
\text { is no longer improving after an injection }\end{array}$ & $\begin{array}{l}\text { Randomized treatment groups included either } \\
\text { prompt (within } 3-10 \text { days after the initial } \\
\text { injection) or deferred (at least } 24 \text { weeks after the } \\
\text { initial injection) focal/grid laser. If deferred, } \\
\text { laser could be added only if edema was still } \\
\text { present } \$ \text { after } 24 \text { weeks and there was no } \\
\text { improvement from two prior consecutive } \\
\text { injections }\end{array}$ \\
\hline $\begin{array}{l}\text { When should focal/grid } \\
\text { laser be repeated after } \\
\text { an initial focal/grid } \\
\text { laser? }\end{array}$ & $\begin{array}{l}\text { Focal/grid laser generally should be repeated at any time that } \\
\text { edema persists or is not improving while giving anti-VEGF } \\
\text { therapy and it is believed that additional benefit will be gained } \\
\text { by repeating laser }\end{array}$ & $\begin{array}{l}\text { Once initiated, focal/grid was re-applied if } 1 \text { ) } \\
\text { edema was present } t^{+}, 2 \text { ) eye did not have } \\
\text { "complete" laser } \$ \text {, and } 3 \text { ) it had been at least } 13 \\
\text { weeks since last laser }\end{array}$ \\
\hline
\end{tabular}

† "Improvement" in the DRCR.net study was defined as "increase of at least 5 letters (approximately 1 line) in visual acuity or at least a $10 \%$ reduction in the central subfield thickness on OCT since the last injection."

FDefined as OCT central subfield thickness $\geq 250 \mu \mathrm{m}$ or edema on clinical exam within $500 \mu \mathrm{m}$ of center of macula or edema $\geq 1$ disc area and within 1 disc area of the center of the macula). macular edema.

DME=Diabetic macular edema, DRCR.net= the Diabetic Retinopathy Clinical Research Network, OCT=Optical coherence tomography, anti$\mathrm{VEGF}=$ anti-vascular endothelial growth factor. 
Table 2

\section{Diabetic Retinopathy Clinical Research Network Intravitreal Injection Technique}

- Pre-injection topical antibiotics can be applied at the discretion of the treating ophthalmologist.

- Confirm and mark the eye for injection.

- Apply topical anesthetic.

- $\quad$ Place the lid speculum.

- Apply povidone iodine directly over and surrounding the injection site (allowing sufficient time for the povidone iodine to dry).

- $\quad$ Locate the injection site $3.0 \mathrm{~mm}$ to $4.0 \mathrm{~mm}$ posterior to the limbus.

- $\quad$ Prepare the proper volume of drug to be injected.

- Inject the drug using a sterile 30-gauge needle into the vitreous cavity pointing toward the optic nerve via the pars plana.

- $\quad$ Remove the lid speculum, avoiding any excess pressure on the eye.

- Assess for any complications and confirm that the central artery is perfused using indirect ophthalmoscopy or confirmation of vision.

- Topical antibiotic can be provided at the discretion of the treating ophthalmologist. 
Table 3

\section{Percent "Success" Following Initial Injections}

\begin{tabular}{cllll}
\hline \multicolumn{5}{c}{ Number of Injections } \\
\hline & $\mathbf{1}$ & $\mathbf{2}$ & $\mathbf{3}$ & $\mathbf{4}$ \\
\hline Ranibizumab +Prompt Laser (N $=180)$ \\
$<250$ CSF thickness & $24 \%$ & $36 \%$ & $45 \%$ & $48 \%$ \\
$\geq 84$ VA letter score & $3 \%$ & $4 \%$ & $9 \%$ & $12 \%$ \\
Either <250 or $\geq 84$ & $25 \%$ & $37 \%$ & $47 \%$ & $51 \%$ \\
\hline
\end{tabular}

Ranibizumab +Deferred Laser $(\mathrm{N}=181)$

$\begin{array}{lllll}<250 \text { CSF thickness } & 25 \% & 31 \% & 44 \% & 40 \% \\ \geq 84 \text { VA letter score } & 3 \% & 8 \% & 7 \% & 7 \% \\ \text { Either }<250 \text { or } \geq 84 & 27 \% & 34 \% & 47 \% & 43 \%\end{array}$

*only includes injections occurring within first 16 weeks

Success $=$ visual acuity letter score 84 or better (approximate Snellen equivalent 20/20 or) better, or optical coherence tomography central subfield thickness less than 250 microns. CSF=Central Subfield, VA= Visual Acuity. 
Table 4

Number of Treatments and Visits During First 2 Years of Intravtireal Ranibizumab Therapy

\begin{tabular}{|c|c|c|c|}
\hline & Visits & Injections & Laser \\
\hline \multicolumn{4}{|l|}{ Year 1} \\
\hline \multicolumn{4}{|c|}{ Ranibizumab+Prompt Laser $(\mathrm{N}=156)$} \\
\hline Maximum & -- & 13 & 4 \\
\hline Median $\left(25^{\text {th }}, 75^{\text {th }}\right.$ quartile $)$ & -- & $8(7,11)$ & $2(1,3)$ \\
\hline Maximal possible per protocol & 13 required per protocol & 13 & 4 \\
\hline \multicolumn{4}{|c|}{ Ranibizumab+Deferred Laser $(\mathrm{N}=161)$} \\
\hline Maximum & -- & 13 & 2 \\
\hline Median $\left(25^{\text {th }}, 75^{\text {th }}\right.$ quartile $)$ & -- & $9(6,11)$ & $0(0,1)$ \\
\hline Maximal possible per protocol & 13 required per protocol & 13 & 2 \\
\hline \multicolumn{4}{|l|}{ Year 2} \\
\hline \multicolumn{4}{|c|}{ Ranibizumab+Prompt Laser $(\mathrm{N}=156)$} \\
\hline Maximum & 14 & 12 & 4 \\
\hline Median (25th, 75th quartile) & $8(5,11)$ & $2(0,4)$ & $0(0,1)$ \\
\hline Maximal possible per protocol & 13 & 13 & 4 \\
\hline \multicolumn{4}{|c|}{ Ranibizumab+Deferred Laser $(\mathrm{N}=161)$} \\
\hline Maximum & 16 & 14 & 3 \\
\hline Median (25th, 75th quartile) & $10(6,12)$ & $3(1,7)$ & $0(0,1)$ \\
\hline Maximal possible per protocol & 13 & 13 & 4 \\
\hline
\end{tabular}


Table 5

Diabetic Retinopathy Clinical Research Network Focal/Grid Laser (Both Focal and Grid Treatment Are Applied When Laser for Diabetic Macular Edema is Considered)

A. Circumstances to Consider

- Edema threatening or involving the center of the macula, including any of the following:

- Optical Coherence Tomography (OCT) central subfield thickness $\geq 2$ standard deviations than the norm (e.g., $\geq 250 \mu \mathrm{m}$ on Stratus OCT)

- Edema within $500 \mu \mathrm{m}$ of the center of the macula

- $\quad$ Edema associated with lipid within $500 \mu \mathrm{m}$ of the center of the macula, or edema $\geq 1$ disc area within 1 disc area of the center of the macula

- $\quad \geq 13$ weeks since focal/grid laser has been given

B. Direct Focal/Grid Treatment

- Direct laser to all microaneurysms in areas of retinal thickening between 500 an $3000 \mu \mathrm{m}$ from the center of the macula

- Direct laser to all microaneurysms in areas of retinal thickening between 300 an $500 \mu \mathrm{m}$ of center of macula if centerinvolved edema persists after an initial session of focal/grid laser, but generally not if the visual acuity is better than $20 / 40$

- $\quad 50 \mu \mathrm{m}$ to $60 \mu \mathrm{m}$ spot size

- $\quad 0.05$ to 0.1 second duration

- Green to yellow wavelengths

- Endpoint of laser burn: change color of microaneurysm or at least a mild gray-white burn evident beneath microaneurysm.

- $\quad$ Grid laser is applied to all areas with edema not associated with microaneurysms between $500 \mu \mathrm{m}$ and $3000 \mu \mathrm{m}$ superiorly, nasally and inferiorly from the center of macula and between $500 \mu \mathrm{m}$ and $3500 \mu \mathrm{m}$ temporally from the center of the macula, separating laser burns at least 2 burn widths apart, again with a $50 \mu \mathrm{m}$ to $60 \mu \mathrm{m}$ spot size and 0.05 to 0.1 second duration. 